\title{
EFEITO DA TEMPERATURA INTERCRÍTICA NO EXPOENTE DE ENCRUAMENTO DE AÇOS BIFÁSICOS*
}

\author{
Aline Silva Magalhães ${ }^{1}$ \\ Suzanny Cristina Soares Martins ${ }^{2}$ \\ Maria Teresa Paulino Aguilar ${ }^{3}$ \\ Wellington Lopes ${ }^{4}$ \\ Elaine Carballo Siqueira Corrêa ${ }^{5}$
}

\section{Resumo}

As elevadas taxas de encruamento nos estágios iniciais de deformação plástica observadas para os aços bifásicos constituem uma das principais características que os habilita para aplicação em componentes estruturais na indústria de automóveis. Neste trabalho foi analisada influência de tratamentos intercríticos com aquecimento em temperaturas diversas, seguido de resfriamento brusco, no comportamento em termos de encruamento de um aço bifásico, por meio de dois métodos distintos de determinação do expoente de encruamento. Observou-se que a elevação da temperatura intercrítica influenciou de modo a reduzir o valor do expoente de encruamento, dado que o aumento da temperatura reflete a elevação do percentual de martensita na microestrutura do aço. Além disso, para as diferentes abordagens aplicadas para o cálculo dos valores de $n$, verificou-se que por meio da técnica de linearização da curva tensão versus deformação foram obtidos valores superiores do expoente de encruamento quando comparada à abordagem utilizando o critério da estricção.

Palavras-chave: Aços bifásicos; Tratamento intercrítico; Expoente de encruamento.

\section{EFFECT OF THE INTERCRITICAL TEMPERATURE ON THE HARDENING EXPOENT OF DUAL PHASE STEELS}

\begin{abstract}
The high work hardening rates in the early stages of plastic deformation observed in the dual phase steel is one of the most important properties that enables them to be used in structural components in the automobile industry. The aim of this work is to analyze the effects of the intercritical heat treatment at various temperatures followed by quenching on the work hardening behavior of the dual phase steel by different methods for determining the strain hardening exponent $n$. It was observed that an increase in the intercritical temperature led to the reduction of the strain hardening exponent value. The phenomenon was associated with the increase of the volume fraction of martensite in the steel microstructure. According to the different approaches applied to calculate $n$ value, upper values were found by the curve linearization technique when compared with the approach using the necking criterion. Keywords: Dual phase steels; Intercritical treatment; Strain hardening expoent.

Eng. de Materiais, Mestre, Professora, DEMAT, CEFET-MG, Belo Horizonte, MG, Brasil.

Eng. de Materiais, Mestre, DEMAT, CEFET-MG, Belo Horizonte, MG, Brasil.

Eng. Metalurgista, Dra., Professora, DEMC, UFMG, Belo Horizonte, MG, Brasil.

Eng. Mecânico, Dr., Professor, DEMAT, CEFET-MG, Belo Horizonte, MG, Brasil.

Eng. Mecânica, Dra., Professora, DEMAT, CEFET-MG, Belo Horizonte, MG, Brasil.
\end{abstract}




\section{INTRODUÇÃO}

A utilização dos aços bifásicos compondo peças estruturais na produção de veículos automotivos se deve à ocorrência de uma favorável combinação de propriedades mecânicas, como elevada resistência à tração, boa ductilidade e alta taxa de encruamento nos estágios iniciais de deformação plástica [1,2,3]. Essas propriedades estão relacionadas à microestrutura das ligas, composta por ilhas de martensita dispersas em uma matriz ferrítica, podendo ainda ser verificada a presença de outros arranjos, como bainita, austenita e carbonetos [4,5]. Nesse contexto, diversas variáveis associadas aos aspectos estruturais dos aços bifásicos influenciam seu comportamento, dentre os quais podem ser destacados: a morfologia, as dimensões e a proporção dos constituintes presentes; sua dispersão; o teor de carbono da martensita e a presença e quantidade de austenita retida [6].

A elevada capacidade de encruamento associada aos aços bifásicos influencia a habilidade desses materiais de exibirem deformação uniforme, ou seja, materiais com alta taxa de encruamento podem ser deformados até magnitude superiores antes de atingir a instabilidade plástica, dando início à estricção. Dessa forma, para processos de fabricação que envolvam deformação plástica, ligas metálicas com esse comportamento são mais atrativas [7,8,9]. Essa alta taxa de encruamento nos aços bifásicos é atribuída basicamente a três fatores: 1) alívio das tensões residuais decorrentes do resfriamento durante a deformação plástica; 2) aumento da densidade de discordâncias na ferrita e 3 ) incompatibilidade plástica entre a ferrita e a martensita, levando ao desenvolvimento de tensões residuais na primeira $[10,11]$. Dessa forma, a elevada taxa de encruamento dos aços bifásicos estaria associada às interações realizadas entre as discordâncias móveis da ferrita com o emaranhado de discordâncias presente na porção deformada plasticamente ao redor da martensita. Ghaheri et al. [12] também relaciona a capacidade de encruamento dos aços bifásicos com a elevada densidade de discordâncias em movimento na matriz ferrítica e a quantidade substancial de tensões residuais. As discordâncias induzidas pela transformação martensítica se moveriam sob tensões de baixa magnitude, gerando tensões de escoamento reduzidas e interagindo entre si, produzindo altas taxas de encruamento $[2,13]$.

O expoente de encruamento, a variável $n$ da Equação de Hollomon $\left(\sigma=k \varepsilon^{n}\right.$, onde é a tensão, $k$ é o coeficiente de resistência e e é a deformação) é considerado um bom indicador do encruamento do material, dado que, quanto maior for o seu valor, maior será a taxa de encruamento, ou seja, maior será a sensibilidade da tensão de acordo com a deformação. Segundo Mazaheri et al. [8], magnitudes elevadas de expoente de encruamento são interessantes em processos de fabricação que envolvem deformação plástica, tornando assim, seu estudo do de significativa relevância em aços para a estampagem.

A determinação do expoente de encruamento pode ser realizada de diferentes formas. Uma primeira abordagem envolve a linearização da curva tensãodeformação reais, considerando o intervalo entre o início do escoamento plástico e a carga máxima no ensaio de tração [14]. O valor de $n$ é dado pelo coeficiente angular da reta obtida a partir de $\log \sigma$ versus $\log \varepsilon$. Outra maneira utilizada para quantificar o encruamento do material é por meio da análise da deformação uniforme real, ou seja, a deformação real até o início da estricção. Segundo Dieter [14], o início da deformação localizada ocorre na carga máxima, na qual a diminuição da área da seção transversal causa uma redução da tensão que, por sua vez, se torna superior ao aumento da capacidade do material de suportar a carga, devido ao encruamento 
Dentro desse contexto, o objetivo deste trabalho foi investigar o comportamento de um aço bifásico submetido a aquecimento intercrítico em temperaturas distintas seguido de resfriamento brusco, no que se refere ao encruamento utilizando a análise do expoente $n$ em abordagens distintas.

\section{MATERIAIS E MÉTODOS}

O material utilizado neste trabalho foi um aço produzido por laminação a frio com espessura de 2,00 mm, cuja composição química é exibida na Tabela 1.

Tabela 1. Composição química (\% em peso) do aço empregado no estudo

\begin{tabular}{lccccccccc}
\hline Elemento & $\mathbf{F e}$ & $\mathbf{C}$ & $\mathbf{M n}$ & $\mathbf{S i}$ & $\mathbf{C r}$ & $\mathbf{N i}$ & $\mathbf{A l}$ & $\mathbf{N b}$ & $\mathbf{W}$ \\
\hline Teor (\%) & $97,4 \%$ & $0,12 \%$ & $1,58 \%$ & $0,49 \%$ & $0,02 \%$ & $0,03 \%$ & $0,05 \%$ & $0,01 \%$ & $0,01 \%$ \\
\hline
\end{tabular}

$\mathrm{Na}$ Tabela 2 são mostrados os parâmetros empregados nos tratamentos térmicos abordando as temperaturas estipuladas, escolhidas a partir da determinação das temperaturas de início e de fim de transformação de fases, para os tratamentos térmicos de normalização (realizado inicialmente no material) e de aquecimento intercrítico com resfriamento rápido (subsequentes à normalização), todos conduzidos em banhos de sal.

Tabela 2. Parâmetros de tratamentos térmicos aplicados ao aço bifásico

\begin{tabular}{ccccc}
\hline Tratamento Térmico & Temperatura & Tempo de encharque & Resfriamento \\
\hline Normalização & $900^{\circ} \mathrm{C}$ & $60 \mathrm{~min}$ & ar \\
\hline $\begin{array}{c}\text { Aquecimento } \\
\text { intercrítico com } \\
\text { resfriamento rápido }\end{array}$ & $730^{\circ} \mathrm{C}$ & & & \\
\cline { 2 - 2 } & $770^{\circ} \mathrm{C}$ & $60 \mathrm{~min}$ & água \\
\hline
\end{tabular}

O expoente de encruamento do material foi obtido a partir dos dados de ensaios de tração, considerando as direções longitudinal e transversal em relação à original de laminação, no intuito de estudar o efeito da direção de laminação anterior ao processamento térmico no comportamento do material. Os ensaios foram conduzidos em uma máquina universal de ensaios Instron modelo 5582 com sistema de controle e aquisição de dados BlueHill 2 e extensômetro acoplado Instron modelo 2630-100, com uma velocidade de movimentação da garra de $4,5 \mathrm{~mm} / \mathrm{min}$. Os corpos de prova para o ensaio de tração, duas amostras para cada condição, foram confeccionados por eletroerosão a fio, em uma máquina Charmiles Modelo Robofil 240, com dimensões de acordo com a norma NBR 6152-1:2002 [15].

A determinação numérica do expoente de encruamento no presente trabalho foi realizada por meio de dois métodos distintos. Uma primeira abordagem envolveu a linearização da curva tensão - deformação reais, considerando o intervalo entre o início da deformação plástica e a carga máxima no ensaio de tração [14]. Nesse caso, o valor de $\mathrm{n}$ foi dado pelo coeficiente angular da reta log $\sigma$ versus log $\varepsilon$. Ressalta-se que os aços bifásicos possuem uma particularidade, inerente aos aços avançados de alta resistência, que é a possibilidade de observação de mais de uma inclinação na reta representativa da curva log tensão versus log deformação $[7,9,16]$. Assim, neste trabalho, além da regressão linear considerando um estágio, foi realizada a análise considerando dois estágios de encruamento. Para essa situação, a determinação do ponto na curva no qual ocorreria a mudança de estágio de encruamento foi realizada com várias simulações, sendo escolhida aquela que levasse à melhor aproximação das retas com os dados reais. 
O segundo método utilizado para a determinação do expoente de encruamento foi a análise da deformação uniforme real,ou seja, a partir do critério da formação da estricção descrito por Dieter (1981) [14]. Especificamente nesta abordagem, foi aplicado para a obtenção dos valores de expoente de encruamento o software Origin versão 8.0, utilizando ainda o recurso de tratamento de dados smoothing, com um parâmetro de ajuste de 25 pontos, com objetivo de evitar uma possível propagação de erros das medidas devido à aplicação de diferenciais durante a análise, suavisando o conjunto de dados [17].

\section{RESULTADOS E DISCUSSÃO}

Os valores de expoentes de encruamento $\mathrm{m}$ médios encontrados por meio da linearização das curvas tensão versus deformação, a partir da análise pela equação de Hollomon, considerando o comportamento do encruamento do material em um estágio, são mostradas na Tabela 3 , para o aço no estado inicial e após os tratamentos intercríticos. Ainda na Tabela 3, são exibidos os valores médios dos coeficientes de determinação $\left(R^{2}\right)$, que medem o ajuste da regressão linear em relação aos valores observados. Quanto mais próximo da unidade maior será a confiabilidade de que o modelo consegue explicar os valores observados [18].

Tabela 3. Expoentes de encruamento do aço bifásico obtidos por meio da análise por Hollomon, considerando o comportamento em um estágio de encruamento

\begin{tabular}{cccc}
\hline \multicolumn{2}{c}{ Condição } & $\mathbf{n}$ & $\mathbf{R}^{\mathbf{2}}$ \\
\hline Estado & $\mathrm{L}$ & 0,1285 & 0,9700 \\
Inicial & $\mathrm{T}$ & 0,1348 & 0,9677 \\
\hline \multirow{2}{*}{$\mathbf{7 3 0} \mathbf{C}$} & $\mathrm{L}$ & 0,2405 & 0,9748 \\
\cline { 2 - 4 } & $\mathrm{T}$ & 0,2478 & 0,9811 \\
\hline \multirow{2}{*}{$\mathbf{7 7 0} \mathbf{C}$} & $\mathrm{L}$ & 0,2256 & 0,9324 \\
\cline { 2 - 4 } & $\mathrm{T}$ & 0,2348 & 0,9411 \\
\hline \multirow{2}{*}{$\mathbf{8 1 0}^{\circ} \mathbf{C}$} & $\mathrm{L}$ & 0,1813 & 0,9221 \\
\cline { 2 - 4 } & $\mathrm{T}$ & 0,2057 & 0,9333 \\
\hline
\end{tabular}

L- longitudinal e T- transversal.

Na Figura 1 são mostrados os valores de expoente de encruamento em função do estado do material - como adquirido ou tratado termicamente, considerando a linearização em um estágio. Avaliando inicialmente os resultados obtidos por meio da linearização, ou seja, pela equação de Hollomon, de maneira geral, considerando um estágio único de encruamento, observa-se uma diminuição dos expoentes de encruamento com o aumento da temperatura intercrítica. Nesse caso, verifica-se que todos os valores referentes ao material submetido a tratamentos térmicos foram superiores ao valor de $n$ para o aço no estado inicial. Avaliando a influência da laminação anterior aos tratamentos, observa-se que a direção do processamento mecânico não resultou em alterações significativas no comportamento do aço.

A Tabela 4 exibe os dados numéricos de expoentes de encruamento $n$ médios obtidos por meio do estudo deste parâmetro considerando dois estágios de encruamento a partir da linearização da curva tensão-deformação, juntamente com os valores médios dos coeficientes de determinação $\left(R^{2}\right)$. 


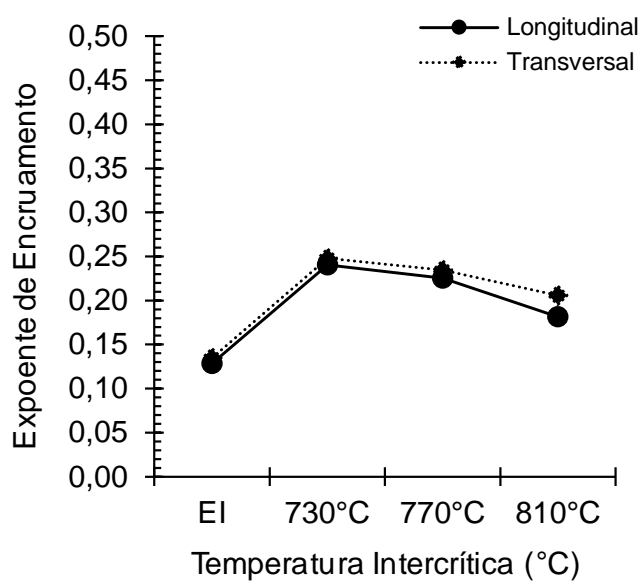

Figura 1. Expoentes de encruamento nas diversas condições estudadas por meio da análise da curva por Hollomon considerando linearização em um estágio. *El - estado inicial.

Tabela 4. Expoentes de encruamento do aço bifásico obtidos por meio da análise por Hollomon, considerando o comportamento em dois estágios de encruamento

\begin{tabular}{cccccc}
\hline \multicolumn{2}{c}{ Condição } & \multicolumn{2}{c}{ Estágio 1 } & \multicolumn{2}{c}{ Estágio 2 } \\
\cline { 2 - 6 } & & $\mathbf{n}$ & $\mathbf{R}^{\mathbf{2}}$ & $\mathbf{n}$ & $\mathbf{R}^{\mathbf{2}}$ \\
\hline \multirow{2}{*}{$\begin{array}{c}\text { Estado } \\
\text { Inicial }\end{array}$} & $\mathrm{L}$ & 0,1836 & 0,9907 & 0,0914 & 0,9954 \\
\hline \multirow{2}{*}{$\mathbf{7 3 0} \mathbf{C}$} & $\mathrm{T}$ & 0,1909 & 0,9953 & 0,0907 & 0,9926 \\
\hline \multirow{2}{*}{$\mathbf{7 7 0} \mathbf{C}$} & $\mathrm{L}$ & 0,3233 & 0,9991 & 0,1670 & 0,9904 \\
\hline \multirow{2}{*}{$\mathbf{8 1 0} \mathbf{C}$} & $\mathrm{T}$ & 0,3069 & 0,9990 & 0,1661 & 0,9939 \\
\cline { 2 - 6 } & $\mathrm{L}$ & 0,3976 & 0,9922 & 0,1371 & 0,9968 \\
\cline { 2 - 6 } & $\mathrm{T}$ & 0,4093 & 0,9933 & 0,1487 & 0,9943 \\
\hline
\end{tabular}

L- longitudinal e T- transversal.

Empregando a análise do encruamento com dois estágios (Figura 2), para o $1^{\circ}$ estágio os valores de $\mathrm{n}$ não parecem seguir uma tendência geral com relação à temperatura intercrítica empregada no tratamento. Para o $2^{\circ}$ estágio, os resultados seguem o mesmo comportamento observado para a avaliação com estágio único, ou seja, a elevação na temperatura levaria a uma queda na capacidade de encruamento do material que, no estado inicial, exibiu expoente de encruamento inferior às demais condições. Novamente, a alteração na direção de laminação não levou a resultados distintos.

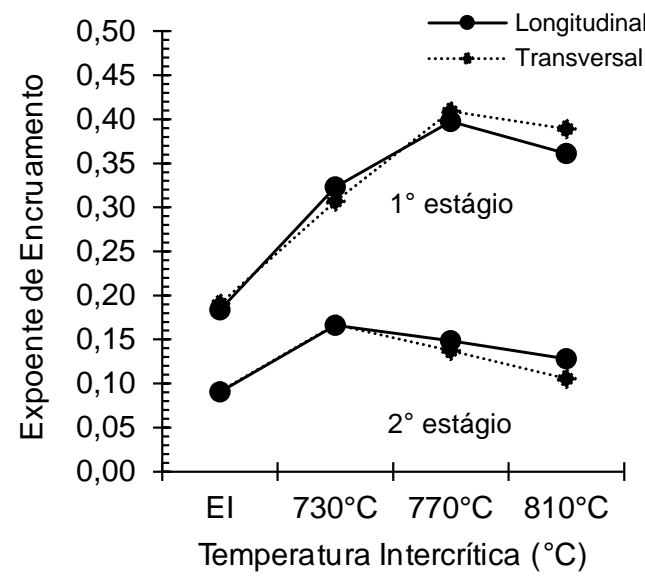

Figura 2. Expoentes de encruamento nas diversas condições estudadas por meio da análise da curva por Hollomon considerando linearização em dois estágios. *El - estado inicial. 
Por fim, os resultados de $\mathrm{n}$ considerando um estágio de encruamento se mostram medianos àqueles observados considerando dois estágios, para todas as condições. Os valores de $\mathrm{R}^{2}$ exibidos na Tabela 3 sugerem que existe uma maior confiabilidade na análise de $n$ quando considerados dois estágios de encruamento, devido ao seu maior módulo para estes casos.

$\mathrm{Na}$ Tabela 5 são apresentados os valores de expoente de encruamento obtidos por meio do critério da formação da estricção.

Tabela 5. Expoentes de encruamento do aço bifásico nas diversas condições estudadas

\begin{tabular}{ccc}
\multicolumn{2}{c}{ Condição } & $n$ \\
\hline Estado & $\mathrm{L}$ & 0,0661 \\
Inicial & $\mathrm{T}$ & 0,0575 \\
\hline \multirow{2}{*}{$\mathbf{7 3 0} \mathbf{C}$} & $\mathrm{L}$ & 0,0934 \\
\cline { 2 - 3 } & $\mathrm{T}$ & 0,0957 \\
\hline \multirow{2}{*}{$\mathbf{7 7 0} \mathbf{C}$} & $\mathrm{L}$ & 0,0713 \\
\cline { 2 - 3 } & $\mathrm{T}$ & 0,0651 \\
\hline \multirow{2}{*}{$\mathbf{8 1 0 ^ { \circ } \mathbf { C }}$} & $\mathrm{L}$ & 0,0511 \\
\cline { 2 - 3 } & $\mathrm{T}$ & 0,0433
\end{tabular}

L- longitudinal e T- transversal.

Para os expoentes de encruamento calculados utilizando o critério da formação da estricção (Figura 3) observa-se, em geral, que os valores são inferiores àqueles obtidos pela linearização da curva tensão-deformação. A elevação da temperatura novamente resultaria em uma diminuição do valor de $n$, assim como na análise por regressão linear considerando um único estágio ou dois de encruamento, neste caso ○ $2^{2}$ estágio.

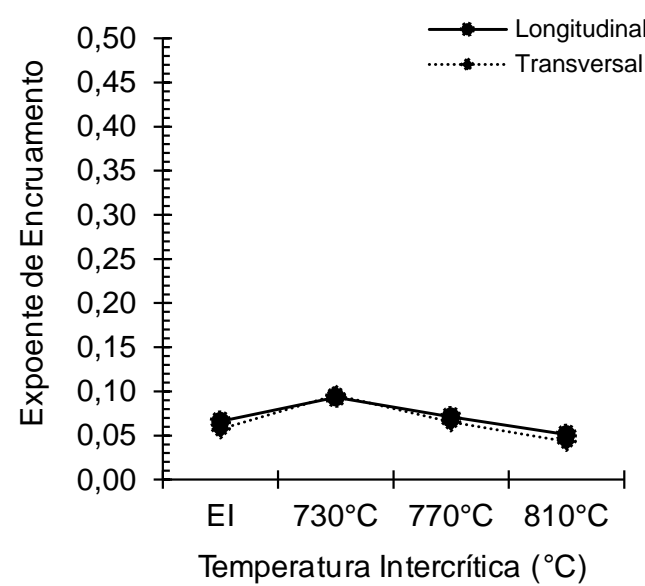

Figura 3. Expoentes de encruamento nas diversas condições considerando o critério da formação da estricção. *El - estado inicial.

Assim, em síntese, nos resultados apresentados considerando tanto a primeira abordagem (em um único estágio ou em dois estágios) como a segunda abordagem para a determinação de $n$, nota-se: i) o expoente de encruamento é reduzido com a elevação da temperatura intercrítica; ii) o material no estado inicial exibe expoente de encruamento inferior às demais condições; iii) os valores de $n$ determinados pela segunda abordagem foram inferiores aos obtidos com a primeira; iv) não foram verificadas diferenças relevantes de acordo com a direção de laminação; v) em relação à primeira abordagem de cálculo, maiores valores de $\mathrm{R}^{2}$ foram observados quando considerados dois estágios de encruamento. 
Considerando a influência da microestrutura no encruamento do aço bifásico, em um trabalho anterior [19], realizado com o mesmo material, foram analisadas as alterações nos microconstituintes em função da temperatura intercrítica de tratamento, nas mesmas condições do presente trabalho. Foi observado que a elevação de temperatura levou ao aumento na fração de martensita e, de forma complementar, resultou na diminuição da fração de ferrita. Já o material no estado inicial apresentou percentuais de ferrita e martensita semelhantes, com uma pequena superioridade para a fração de martensita.

Movahed et al. (2009) [9] ao estudar o comportamento de um aço bifásico 0,11\%C$0,53 \% \mathrm{Mn}$, por meio da linearização da equação de Hollomon, consideraram a existência de apenas um ou de dois estágios de encruamento, de acordo com a temperatura intercrítica de processamento. Em contraste com os resultados exibidos para os aços investigados no presente trabalho, observaram uma elevação dos valores de $\mathrm{n}$ com o aumento da temperatura e, consequentemente, com a fração volumétrica de martensita formada. O comportamento verificado foi relacionado à diferença de resistência mecânica entre a ferrita e a martensita com a elevação da proporção do último constituinte, que afetaria o encruamento do material.

Mazaheri et al. $(2014,2014)$ [7,8], empregando a linearização da equação de Hollomon e considerando o encruamento de um aço bifásico $0,17 \% \mathrm{C}-1,15 \% \mathrm{Mn}$ em dois estágios, observaram que, similarmente aos resultados exibidos no presente trabalho para ambos os materiais, o $2^{\circ}$ estágio de encruamento seria caracterizado pela diminuição dos valores de $\mathrm{n}$ com a temperatura de aquecimento no tratamento intercrítico. Em relação ao $1^{\circ}$ estágio, também não verificaram uma tendência clara de comportamento do expoente de encruamento com a temperatura mencionada. Nesse caso, contrastando com os resultados do presente trabalho, os valores de $n$ do $1^{\circ}$ estágio foram inferiores aos do $2^{\circ}$ estágio. Segundo os autores, inicialmente, correspondendo ao $1^{\text {o }}$ estágio de encruamento, a deformação plástica ocorreria essencialmente na ferrita, ficando a martensita sujeita apenas à deformação elástica. No $2^{\circ}$ estágio, ambos constituintes deformariam plasticamente, porém em magnitudes distintas.

Lai et al (2015) [20], aplicando o método de análise do expoente de encruamento por meio do critério da formação da estricção em um aço com microestrutura bifásica, ferrita e martensita, submetido a tratamento intercrítico em duas condições distintas, observaram que a elevação da temperatura resultou em um aumento do volume de martensita na estrutura que, por sua vez, influenciou de forma a elevar o expoente de encruamento do material.

No caso de elevação da temperatura intercrítica de tratamento térmico, implicando no aumento da fração volumétrica de martensita na estrutura bifásica, em detrimento do teor de ferrita, o teor de carbono dessa segunda fase seria reduzido. Dessa forma, a resistência mecânica da martensita diminuiria e, com isso, sua plasticidade aumentaria. Como consequência final, o expoente de encruamento também seria reduzido, conforme os resultados exibidos no presente trabalho, independente da abordagem por meio da qual o valor de $\mathrm{n}$ foi determinado.

\section{CONCLUSÃO}

- Considerando tanto a abordagem a partir da linearização da curva tensão deformação como o critério da formação da estricção, foi observado que o expoente de encruamento $\mathrm{n}$ da equação de Hollomon é reduzido com a elevação da temperatura intercrítica e que, o aço no estado inicial exibiu expoente de 
encruamento inferior às demais condições.

- Para as análises do expoente de encruamento considerando estágio único e dois estágios de encruamento verificou-se que os valores obtidos no estágio único foram intermediários aos valores mostrados em dois estágios e ainda que os resultados sugerem que existe uma maior confiabilidade na análise desse expoente quando considerados dois estágios de encruamento.

- Ao comparar as duas abordagens utilizadas para ao cálculo do expoente de encruamento, verificou-se que os valores obtidos pelo critério da formação da estricção foram inferiores àqueles observados a partir da linearização da curva tensão - deformação.

\section{Agradecimentos}

Os autores agradecem à CAPES pelas bolsas de mestrado e à MetalTemper pelos tratamentos térmicos.

\section{REFERÊNCIAS}

1 Wang, W.; Wei, X. The effect of martensite volume and distribution on shear fracture propagation of 600-1000 MPa dual phase sheet steels in the process of deep drawing. International Journal Mechanical Science, 2013; 67: 100-107.

2 Maleque, M.A.; Poon, Y.M.; Masjuki. H.H. The effect of intercritical heat treatment on the mechanical properties of AISI 3115 steel. Journal of Materiais Processing Techonoly,2004, 153-154, 482-487.

3 Wang, W.; He, C.; Zhao, Z.; Wei, X. The limit drawing ratio and formability prediction of advanced high strength dual-phase steels. Materials and Design, 2011, 32, 3320-3327.

4 Seydrezai, H.; Pilkey, A. K.; Boyd, J. D. Effect of pre-IC annealing treatments on the final microstructure and work hardening behavior of a dual-phase steel. Materials and Science Engineering A, v. 594, p. 178-188, 2014.

5 Ghassemi-Armaki, H.; Maab, R.; Bhat, S. P.; SRIRAM, S.; GREER, J. R.; KUMAR, K. S. Deformation response of ferrite and martensite in dual-phase steel. Acta Materialia, v. 62, p. 197-211, 2014.

6 Kuziak, R.; Kawalla, R.; Waengler, S. Advanced high strength steels for automotive industry. Archives of civil and mechanical engineering, v. 8, n. 2, p. 103-117, 2008.

7 Mazaheri, Y.; Kermanpur, A.; Najafizadeh, A. A novel route for development of ultrahigh strength dual phase steels. Materials Science and Engineering A, v. 619, p. 1-11, 2014.

8 Mazaheri, Y.; Kermanpur, A.; Najafizadeh, A; Saeidi, N. Effects of initial microstructure and thermomechanical processing parameters on microstructures and mechanical properties of ultrafine grained dual phase steels. Materials Science and Engineering $A$, v. 612, p. 54-62, 2014.

9 Movahed, P.; Kolahgar, S.; Marashi, S.P.H.; Pouranvari, M.; Parvin, N. The effect of intercritical heat treatment temperature on the tensile properties and work hardening behavior of ferrite-martensite dual phase steels sheets. Materials Science and Engineering A. 2009; 518:1-6.

10 Gorni, A. A.; Branchini, O. L. G. A produção de chapas de aço bifásico. Máquinas e Metais, p. 84-89, 1989.

11 Fereiduni, E.; Banaddkouki, S. S. G. Ferrite hardening response in a low alloy ferritemartensite dual phase steel. Journal of Alloys and Compounds, 2014, 589,288-294.

12 Ghaheri, A.; Shafyei, A.; Honarmand, M. Effects of inter-critical temperatures on martensite morphology, volume fraction and mechanical properties of dual-phase steels obtained from direct and continuous annealing cycles. Materials and Design. 2014; 62: 305-319. 
13 Hayat, F.; Uzun, H. Effect of heat treatment on microestructure, mechanical properties and fracture behavior of ship and dual phase steels. Journal of Iron and Steel Research, 2011, 18, 65-72.

14 Dieter, G. E. Mechanical metallurgy. 3. Columbus: McGraw-Hill, 1981.

15 Correa, S. M. B. B. Probabilidade e estatítica. 2. Belo Horizonte: PUC Minas Virtual, 2003.

16 Asm Handbook. Properties and selection irons steels and high performance alloys. Ohio: ASM International, 1993.

17 Zhao, Z.; Tong, T.; Liang, J.; Yin, H.; ZHAO, A.; TANG, D. Microstructure, mechanical properties and fracture behavior of ultra-high strength dual-phase steel. Materials Science \& Engineering. 2014; 618, 182-188

18 Correa, E. C. S. Aspectos do encruamento de metais previamente deformados a frio [tese]. Belo Horizonte (MG): Departamento de Engenharia Metalúrgica e de Materiais da UFMG, 2004

19 Correa, S. M. B. B. Probabilidade e estatítica. 2. Belo Horizonte: PUC Minas Virtual, 2003.

20 Magalhães, A. S.; Martins, S. C. S.; Pinto, M. F.; Aguilar, M. T. P.; Costa, M. C. M.S.; Lopes, W.; Corrêa, E. C. S. Influência da temperatura intercrítica na microestrutura e nas propriedades mecânicas de um aço bifásico. "In": Associação Brasileira de Metalurgia, Materiais e Mineração. $70^{\circ}$ Congresso Anual da ABM: Anais do $70^{\circ}$ Congresso Anual da ABM; 17 a 21 de agosto de 2015; São Paulo. São Paulo: ABM; 2015. p.1-10.

21 Lai, Q., Brassart, L.; Bouaziz, O.; Goune, M.; Verdier, M.; Parry, G.; Perlade, A.; Brechet, Y.; Pardoen, T. Influence of martensite volume fraction and hardness on the plastic behavior of dual-phase steels: Experiments and micromechanical modeling. International Journal of Plasticity. 2015, 1-17. 